\title{
Linx
}

Revue des linguistes de l'université Paris X Nanterre

$53 \mid 2005$

Le semi-figement

\section{Sur les constructions causatives figées du français}

\section{Christian Molinier}

\section{(2) OpenEdition \\ Journals}

Édition électronique

URL : http://journals.openedition.org/linx/284

DOI : $10.4000 /$ linx.284

ISSN : 2118-9692

\section{Éditeur}

Presses universitaires de Paris Nanterre

\section{Édition imprimée}

Date de publication : 1 décembre 2005

Pagination : 197-216

ISSN : 0246-8743

\section{Référence électronique}

Christian Molinier, «Sur les constructions causatives figées du français », Linx [En ligne], 53 | 2005

mis en ligne le 15 février 2011, consulté le 01 mai 2019. URL : http://journals.openedition.org/linx/284 ; DOI : 10.4000/linx.284 


\title{
Sur les constructions causatives figées du français
}

\author{
Christian Molinier \\ Université de Toulouse-Le Mirail et ERSS-CNRS
}

\section{Introduction}

Les dictionnaires et les glossaires du français répertorient au moins 130 expressions verbales en faire, dans lesquelles le verbe faire régit un verbe à l'infinitif et s'analyse comme un opérateur causatif. Les expressions sont du genre de celles qui figurent dans les phrases suivantes: Jean fait marcher Léa, Cette histoire fait jaser, Léa fait bouillir la marmite, Max a fait mourir Léa à petit feu, Max s'est fait secouer les puces par Luc, Le succès se fait attendre, etc.

Ces phrases peuvent être considérées comme figées à des degrés variables en fonction tout d'abord du degré d'autonomie de la phrase élémentaire vis-à-vis de l'opérateur causatif $N$ faire \#. Ainsi, Léa a marché, On va jaser, On lui a secoué les puces sont des phrases parfaitement acceptables, avec le sens qu'elles ont dans la structure causative, même si leur emploi est plus fréquent dans la structure causative. A l'inverse, dans les phrases Léa fait bouillir la marmite ou Max a fait mourir Léa à petit feu, il parait plus difficile de dissocier les phrases La marmite bout ou Léa est morte à petit feux de l'opérateur causatif, et de considérer qu'elles sont porteuses d'une interprétation particulière entrant en combinaison avec celle de l'opérateur causatif. Par ailleurs, dans ces phrases considérées globalement, on peut observer soit le figement de tout le groupe verbal à l'infinitif avec le verbe faire, comme dans Léa fait bouillir la marmite ou Marie fait danser l'anse du panier, soit le figement d'une partie seulement du groupe verbal à 
l'infinitif avec le verbe faire, la position unique de complément, ou l'une des positions de complément, restant libre, comme dans Max fait marcher Léa et Max a fait entendre raison à Léa.

Dans notre classification générale des phrases figées en faire, inspirée des travaux de M. Gross sur le lexique-grammaire du français, nous désignons cette classe sous l'intitulé $F V$. Sa structure définitionnelle est :

$$
\text { No faire } V W
$$

et, plus spécifiquement :

$$
N_{0}\left(N_{0}=: N \text { bum }+N \text {-bum) faire } V \text {-inf } N_{1}\left(E+\text { Prép } N_{2}\right)(E+A d v)\right.
$$

Nous étudierons essentiellement ici la structure syntaxique de ces phrases, en recourant systématiquement à la comparaison avec la structure des phrases libres.

\section{Le sujet $N_{0}$}

Le sujet des expressions verbales figées de notre classe FV est soit un Nbum soit un $N$-bum $\left(N_{0}=: N \text { bum }+N \text {-bum }\right)^{1}$. Son rôle sémantique est tout à fait semblable à celui du sujet de l'opérateur causatif $N$ faire \# appliqué à une phrase élémentaire libre (M. Gross 1981). Ainsi, dans l'exemple (1), où l'on a $N_{0}=:$ Nhum:
a. Max a fait \# Léa travailler
b. Max a fait travailler Léa

le sujet est agentif. Il intervient auprès du sujet humain de la phrase élémentaire pour lui imposer un comportement ou une action (cf. L. Tasmowski 1985 : 339). Dans l'exemple (2), où l'on a $N_{0}=: N$-bum:
a. La pluie a fait \# Le terrain glisser
b. La pluie a fait glisser le terrain

le sujet est causatif. Il désigne le phénomène qui est à l'origine du processus ou qui a provoqué le processus décrit dans la phrase élémentaire.

Dans les exemples suivants, où $N_{0}=:$ Nbum, $N_{0}$ est dépourvu de toute interprétation agentive et l'opérateur causatif a pour seul effet de conférer une sorte de voix passive à la phrase élémentaire. $N_{0}$, coréférent au C.O.D. ou au C.O.I. de la phrase élémentaire, hérite du rôle sémantique qui est celui du C.O.D. ou du C.O.I. dans celleci. Ainsi, dans (3), $N_{0}$ est un patient comme le C.O.D de la phrase élémentaire :

(3) a. Max a fait \# Le directeur réprimander Max

b. Max s'est fait réprimander par le directeur

\footnotetext{
${ }^{1}$ Cependant, quelques expressions (faire suer Nbum, faire chier Nbum, faire dresser les cheveux sur la tête à Nhum, faire marrer Nhum) admettent un sujet non restreint (soit Nhum, soit $N$-bum, soit $Q u P$ ) et expriment un sentiment qui affecte le Nhum complément. Sur la base de ces deux propriétés, ces expressions pourraient figurer dans la classe F5 aux côtés de faire plaisir à Nhum, faire envie à Nhum, etc.
} 
et dans (4), No est un bénéficiaire, comme le C.O.I. de la phrase élémentaire :

(4) a. Max a fait \# Le directeur reprocher à Max son incompétence

b. Max s'est fait reprocher son incompétence par le directeur

D'un point de vue sémantique, le rôle de l'opérateur causatif revient essentiellement ici à placer en position de topique l'un des arguments de la phrase élémentaire. Cependant, à toute phrase élémentaire dont l'un des arguments est C.O.D. ou C.O.I. ne peut s'associer une phrase à opérateur causatif ayant pour rôle de topicaliser l'un de ces arguments. Ainsi, si $N_{1}$ le C.O.D. de la phrase élémentaire, n'est pas un vrai patient, i.e. un Nhum affecté par le processus décrit, alors il ne peut pas être topicalisé au moyen de l'opérateur causatif à fonction de topicalisation :

Max a fait \# De nombreuses personnes ont aperçu Max

* Max s'est fait apercevoir par de nombreuses personnes

VS

Max a fait \# De nombreuses personnes ont abordé Max

Max s'est fait aborder par de nombreuses personnes

Remarque. Le verbe voir a des emplois semblables à celui de l'opérateur causatif dans cette dernière fonction :

Max s'est vu expulser de la salle par les vigiles

Max s'est vu confier une mission délicate par le directeur

Nous retrouvons dans les phrases figées de type $N_{0}$ faire $V W$ du français, pour le sujet $N_{0}$ de l'opérateur causatif, les mêmes valeurs que pour celui des phrases libres :

Léa fait marcher la boutique

Ce fait divers a fait couler beaucoup d'encre

Max s'est fait trouer la peau

\section{Les types de constructions en faire $V$-inf $W$}

\subsection{Les constructions libres}

Dans les constructions libres $N_{0}$ faire $V$-inf $W$, il convient de distinguer, comme le font Damourette et Pichon (1911-1950, t. III $\$ 1107$ et t. V $\ 2046)$, deux types de construction, la seconde pouvant à son tour se subdiviser en deux sous-types. Le premier type de construction - désormais construction de type 1 - peut être représenté par les exemples :

(1) Je le ferai criembre ${ }^{2}$

(2) Je le ferai chanter cette chanson ${ }^{3}$

2 criembre en ancien français signifie « éprouver de la crainte », « avoir peur ». 
Le verbe faire est ici suivi d'une véritable proposition infinitive, comparable à celle de la phrase latine Jussit Trimarchiden facere aestimationem. Cette proposition infinitive est formée d'un C.O.D. de faire (le pronom préverbal = : le en (1) et (2), le GN = : Louis en (3)), accompagné d'un groupe verbal à l'infinitif « attribut» du C.O.D.

Le second type de construction se caractérise par la «coalescence» entre le verbe faire et l'infinitif placé immédiatement à sa droite. Deux sous-types sont distingués, le premier - désormais construction de type $2 \mathrm{a}$ - peut être représenté par les exemples :

(4) Je lui ferai criembre 4

(5) Je lui ferai chanter une chanson ${ }^{5}$

le second - désormais construction de type $2 \mathrm{~b}$ - peut être représenté par l'exemple :

(6) Je ferai chanter une chanson par Louis

Dans les constructions de type 2 , les arguments de la phrase élémentaire acquièrent un nouveau statut par rapport au complexe verbal faire $V$-inf. En (4) et (5), dans la construction de type 2a, les compléments datifs désignent l'individu «intéressé » par le procès global (Damourette et Pichon, $\mathbb{\$} 1080$ ) et sont syntaxiquement liés au verbe faire. En (6), dans la construction de type 2b, le complément d'agent désigne l'« agent d'exécution » du procès global (Damourette et Pichon, \1080) et est syntaxiquement lié au verbe infinitif (Damourette et Pichon, $\$ 1599)^{6}$.

\footnotetext{
${ }^{3}$ Cette construction est aujourd'hui considérée comme vieillie mais on la rencontre parfois. On lui préfère la construction Je lui ferai chanter cette chanson. Dans cette construction, le C.O.D. de chanter peut être pronominalisé au moyen d'un pronom préverbal antéposé au verbe chanter: Je le ferai la chanter, ce qui tend à montrer la relative autonomie de $V$-inf par rapport à faire.

${ }^{4}$ Cette construction appartient à l'ancienne langue. Mais il n'est pas rare de la rencontrer aujourd'hui, en particulier lorsque le verbe infinitif régit un complément indirect, cf. Blanche lui faisait penser à une grosse poupée, R. Sabatier, Trois sucettes à la menthe, in M. Grevisse 1975, \1008; Je lui ferai changer d'opinion, M. H. 06/02/04.

${ }^{5}$ Damourette et Pichon ( $(1107)$, qui désignent la construction de type 1 sous l'étiquette de tour direct et la construction de type 2 a sous l'étiquette de tour assomptival, considèrent contre Tobler et Meyer-Lübke et avec Ronjat, qui cite le vers 4 de la Cantilène de Sainte Eulalie : Voldrent la faire diavle servir, que le tour direct est le plus ancien.

${ }^{6}$ Les interprétations respectives des constructions $2 \mathrm{a}$ et $2 \mathrm{~b}$ ont donné lieu à de nombreuses discussions. Pour Damourette et Pichon (\$2047), dans la phrase Le chirurgien fit opérer ce malade par son interne, c'est le processus qui est au premier plan et « l'agent de l'opération est un point accessoire ». Dans la phrase Le chirurgien fit opérer ce malade à son interne, « la fin principale que l'on envisage ..., c'est de faire que l'interne opère, c'est-à-dire de lui donner l'occasion de perfectionner sa virtuosité chirurgicale ». Pour M. Herslund (1986 : 239), la construction 2b fait du patient de la prédication secondaire (Dans l'exemple de D. \& P. Le chirurgien opérer le malade) le centre d'intérêt et lui accorde une thématicité plus grande, tandis que la construction $2 \mathrm{a}$ fait de l'agent le centre d'intérêt et lui accorde une thématicité plus grande.
} 


\subsection{Les constructions figées}

Ces distinctions valent aussi pour les constructions figées $N_{0}$ faire $V$-inf $W$. Cependant, pour les constructions figées, la situation est modifiée du fait que la phrase prend généralement un sens global non réductible à celui d'un opérateur causatif appliqué à une phrase élémentaire et que le complexe faire $V$-inf tend à s'assimiler, sémantiquement, dans tous les types distingués, à un verbe unique pourvu de ses propres arguments. Dans les phrases figées, pour les deux types de construction, $V$-inf est beaucoup plus étroitement lié au verbe faire. Considérons la phrase (7), dans son sens idiomatique « exercer un chantage sur quelqu'un » :

(7) Je ferai chanter Louis

" J'exercerai un chantage sur Louis »

Le $V$-inf $=$ : chanter figure obligatoirement à droite du verbe faire et ne peut en aucun cas être précédé du $G N=$ : Louis, dans quelque variété de français que ce soit :

(8) * Je ferai Louis chanter

" J'exercerai un chantage sur Louis »

Par ailleurs, faire chanter est perçu comme un prédicat sémantique unique, muni de deux arguments, un agent et un patient. Considérons à présent la phrase (3), dans son sens littéral :

(3) Je ferai chanter Louis

Dans certaines variétés de français contemporain (français de la Guyane, des Antilles, de la Réunion), ainsi qu'en français classique, on rencontre couramment, parallèlement à (3) :

\section{Je ferai Louis chanter}

De même, alors qu'au sens littéral, et ce dans toutes les variétés de français, on dit aussi bien (9) que (10) :

(9) Paul a laissé Marie tomber

(10) Paul a laissé tomber Marie

le sens idiomatique «abandonner » n'est possible que dans le cas où $V$-inf $=$ : tomber figure directement à droite du verbe laisser ${ }^{7}$.

\section{Sous-classification des phrases figées No faire $V$-inf $W$}

Nous présentons sous forme de table l'ensemble des phrases figées de la classe $F V$. Ces phrases sont segmentées en une suite linéaire de champs dont l'intitulé précise la nature. Le codage de la nature du sujet de $V$-inf permet de distinguer trois gran-

${ }^{7}$ Cette dernière remarque, due à N. Ruwet, est rapportée par L. Tasmowski 1985. 
des sous-classes de phrases figées $F V$, qui correspondent aux constructions 1, 2a, et 2b spécifiées en fonction des analyses de Damourette et Pichon.

\subsection{Les phrases figées de structure No faire $V^{1}$-inf $W$}

Les phrases figées $N_{0}$ faire $V^{1}$-inf $W$ sont celles dont le sujet de $V$-inf est le $G N$, libre ou figé, placé normalement à droite de $V$-inf et noté $N_{1}$. Ces phrases sont donc du type On a fait chanter Louis ou Léa fait bouillir la marmite. Les phrases $N_{0}$ faire $V^{1}$-inf $W$ sont les plus nombreuses (72 sur les 132 de notre corpus). Plusieurs cas sont à distinguer pour le sujet $N_{1}$ de $V^{1}$-inf $W$ :

- Le sujet est fixe, il est figé avec $V$-inf dans l'expression verbale. Ce cas est le plus fréquent:

Léa fait bouillir la marmite

Léa fait bruire ses fuseaux

Léa fait danser l'anse du panier

Léa fait durer le plaisir

Léa fait jouer la corde sensible

Léa a fait sauter les scellés

etc.

- Le sujet est un groupe nominal libre humain $\left(N_{1}=: N-\right.$ bum $)$ :

Max fait chanter Luc

Max fait damner ses parents

Max fait marcher Luc

Max a fait mourir Luc à petits feux

Max fait suer le burnous

etc.

ou un groupe nominal libre non humain $\left(N_{1}=: N\right.$-bum $)$ :

Max fait marcher (la boutique + l'usine + la ferme $+\ldots$ )

La pluie a fait périr (les plantes + les arbres + les fleurs $+\ldots$ )

etc.

- Le sujet correspond au pronom réfléchi se, coréférent au sujet $N_{o}$ de faire et placé à gauche de faire. Dans ce cas, la position $N_{1}$ de la table est vide :

Max se fait

bier

Max ne se fait

pas chier

Max se fait

pousser

etc. 

suivantes :

- Le sujet, non exprimé, est indéfini. Considérons à titre d'exemples les phrases

(1) Cette histoire fait jaser

(2) Max fait parler de lui

(3) On fait aller

En (1), le sujet de jaser, non exprimé, est un sujet humain à référence arbitraire. On peut le rapprocher d'un GN générique tel que les gens: en effet, parallèlement à Cette histoire fait jaser, on accepte, avec le même sens Cette histoire fait jaser les gens.

Dans la phrase (2), le sujet non exprimé est également un sujet humain à référence arbitraire, mais l'introduction d'un GN générique tel que les gens est impossible ici, en raison vraisemblablement de la présence du complément indirect de lui:

? Max fait parler les gens de lui

? Max fait parler de lui les gens

Dans la phrase (3), le sujet de aller semble être assimilable au pronom démonstratif non humain ça. En effet, (3) s'associe naturellement à la question stéréotypée Comment ça va?

$N_{1}$ sujet peut être cooccurrent avec un complément indirect à $N_{2}\left(N_{2}=\right.$ : Nhum). $N_{2}$ représente alors l'individu affecté par le procès :

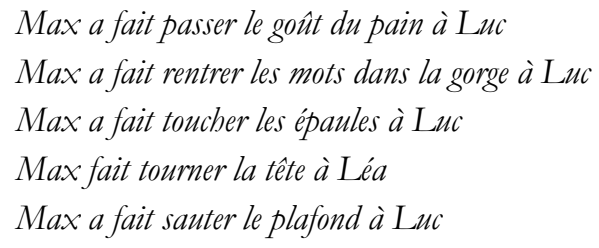

et $N_{1}$ est souvent lié à $N_{2}$ par une relation partie-tout, comme dans quatre des cinq exemples ci-dessus.

Du point de vue du fonctionnement syntaxique, pour les phrases relevant de la structure $N_{0}$ faire $V^{1}$-inf $W$, il n'existe aucune différence entre phrases libres et phrases figées, notamment pour la passivation. Ainsi, lorsque $V$-inf est un inergatif ${ }^{8}$, la passivation de la phrase, avec placement de $N_{1}$ en position sujet, est totalement interdite, qu'il s'agisse de phrases libres :

$$
\begin{aligned}
& \text { Max a fait travailler Luc } \\
& ={ }^{*} \text { Luc a été fait travailler par Max }
\end{aligned}
$$

ou de phrases figées :

\footnotetext{
${ }^{8}$ Dans la tradition générativiste, on désigne sous le nom d'inaccusatifs des verbes intransitifs tels que arriver, venir, tomber, parvenir, etc., qui n'assignent pas de rôle thématique au sujet, et dont le sujet est engendré dans le $V P$, à la manière d'un C.O.D. Les inaccusatifs s'opposent aux inergatifs, tels que parler, dormir, travailler, téléphoner, etc., qui assignent un rôle thématique à leur sujet et sont les vrais intransitifs.
} 


\author{
Max a fait marcher Luc \\ «Max a berné Luc » \\ $=\quad{ }^{*}$ Luc a été fait marcher par Max
}

Mais elle est beaucoup mieux acceptée lorsque $V$-inf est un inaccusatif, qu'il s'agisse indifféremment de phrases libres :

On a fait parvenir ce message à Luc

$=\quad$ ?Ce message a été fait parvenir à Luc

ou de phrases figées :

$$
\begin{aligned}
& \text { On a fait venir Luc à Jubé } \\
& =\quad \text { ?Luc a été fait venir à Jubé } \\
& \text { On a fait mourir Luc à petits feux } \\
& =\quad \text { ?Luc a été fait mourir à petits feux }
\end{aligned}
$$

Il apparaît donc que l'unité sémantique que semble manifester faire Vinf dans les expressions figées (cf. faire marcher, "berner ») ne permet pas de conférer le statut de C.O.D. au GN qui suit. Celui-ci demeure, dans les cas observés ci-dessus, le sujet d'un verbe inaccusatif ou d'un verbe inergatif et fonctionne comme tel.

On rappelle pour mémoire qu'en italien, en occitan, et en français jusqu'au $17^{\mathrm{ème}}$ siècle, la passivation est parfaitement courante dans les phrases de structure $N_{0}$ faire $V^{1}$-inf $W$ lorsque $V$-inf est un inaccusatif :

Italien

Hanno fatto (morire + venire) Giovanni

$=$ Giovanni è stato fatto (morire + venire)

Occitan

An fach (morir + venir) Max

$=\quad$ Max es estat fach (morir + venir)

Français classique

On fit (mourir + venir) Max

$=\quad$ Max fut fait (mourir + venir $)^{9}$

\footnotetext{
${ }^{9}$ Nombreux exemples dans A. Haase (1965: 171) de phrases du type il fut fait mourir, il fut fait venir, il fut fait tomber, etc. Selon Vaugelas (1647: 251), « cette façon de parler (il fut fait mourir) est toute commune le long de la rivière Loire, et dans les provinces voisines, pour dire il fut exécuté à mort. La noblesse du pays l'a apportée à la cour, où plusieurs le disent aussi, et M. Coeffeteau qui était de la province du Maine, en a usé toutes les fois que l'occasion s'est présentée. Les italiens ont cette même phrase, et le cardinal Bontivoglio, l'un des plus exacts et des plus élégants écrivains de toute l'Italie, s'en est servi en son histoire de la guerre de Flandre au quatrième livre, Lo Strale, dit-il, già Borgomastro d'Anversa, e che tanto haveva fomentato seditioni di quellà città, fu fatto morire in Vivorde. ... Valencia, dit-il, cade in potere degli Ugonotti, i quali ne sono fatti uscir poco dopo ... Nous n'avons point encore étendu cette
} 


\subsection{Les phrases figées de structure No faire $V^{2}$-inf $W$}

Les phrases figées de structure $N_{0}$ faire $V^{2}$-inf $W$ sont beaucoup moins nombreuses que les phrases figées de structure $N_{0}$ faire $V^{1}$-inf $W$ (21 sur les 132 de notre corpus). Le sujet de $V$-inf, $N_{2}$, figure à titre de complément prépositionnel en $a$, et il s'agit d'un $N$ bumain $\left(N_{2}=:\right.$ Nhum). $N_{2}$ est nécessairement cooccurrent à un complément d'objet direct de $V$-inf, $\left(N_{1}\right)$.

Ces phrases relèvent de la construction $2 \mathrm{a}$, et pour elles, toute autre construction est interdite. Ainsi, dans le cas de la structure libre, les phrases suivantes relevant des constructions $1,2 \mathrm{a}$ et $2 \mathrm{~b}$ sont toutes trois acceptées avec les nuances de sens et de registre signalées plus haut :

Je le ferai chanter une chanson

Je lui ferai chanter une chanson

Je ferai chanter une chanson par lui

L'expression verbale faire chanter une autre chanson «faire baisser le ton, faire adopter un comportement moins agressif» (Rey-Chantreau 1979) relève de la construction $2 \mathrm{a}$ et est incompatible avec une autre construction :

* Je le ferai chanter une autre chanson

Je lui ferai chanter une autre chanson

* Je ferai chanter une autre chanson par lui

De même, si les phrases suivantes sont acceptées avec la nuance «langue vieillie » ou « langue classique » :

Elle le fit (boire + avaler) son médicament

la phrase suivante dans laquelle avaler la pilule signifie «supporter un désagrément, une insulte ... » (Furetière) n’est compatible qu'avec la construction 2a :

*Elle le fit avaler la pilule

Elle lui fit avaler la pilule

On peut distinguer deux sous-classes de phrases figées $N_{0}$ faire $V^{2}$-inf $W$ en fonction de la nature du complément d'objet direct de $V$-inf:

- Le complément d'objet direct occupe la position $N_{1}$, le plus souvent il est figé avec $V$-inf:

Max a fait avaler la pilule à Léa

Max a fait baisser la chanterelle à Léa

Max a fait danser la carmagnole à Luc

locution fut fait mourir, comme font les italiens à d'autres phrases semblables. Mais nonobstant tout ce que je viens de dire, qui semblerait suffisant pour l'autoriser, il est certain qu'elle est condamnée de tous ceux qui font profession de bien parler et de bien écrire. » 
Max a fait entendre raison à Luc

Max a fait passer un mavvais quart d'beure à Luc

Max a fait perdre le goût du pain à Luc

Max a fait avaler (ce bobard + cette bistoire + Qu P) à Luc

etc.

- Le complément d'objet direct est un pronom préverbal placé à gauche de faire. Dans ce cas, dans la table, la position $N_{1}$ demeure vide en général :

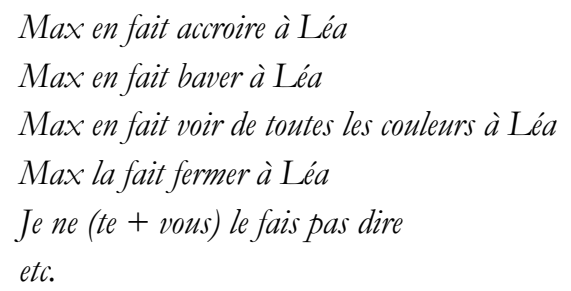

Dans les trois premiers exemples, le pronom en a une interprétation indéfinie. Il pronominalise un GN non humain générique du type des choses. Dans l'exemple Max en fait voir de toutes les couleurs à Léa, de toutes les couleurs, que nous avons fait figurer dans la position $N_{1}$, est un élargissement attributif du C.O.D. en, comme douce dans Max se la coule douce. Dans Max la fait fermer à Léa, la pourrait pronominaliser la gueule, les phrases Max la fait fermer à Léa et Max fait fermer sa gueule à Léa étant synonymes : le pronom la jouerait un rôle euphémique par rapport au GN. Les phrases formulaires Je ne (te + vous) le fais pas dire supposent une situation d'interlocution, mais on peut les trouver à la troisième personne, dans le cas d'un locuteur parlant de lui à la troisième personne (e.g. Alain Delon): Il ne vous le fait pas dire, ou dans le cas du style indirect libre: Il ne le lui faisait pas dire, ou même à la deuxième personne dans le type d'interlocution adopté par M. Butor dans La modification: Tu ne me le faisais pas dire.

Tout comme dans le cas des phrases relevant de la structure $N_{0}$ faire $V^{1}$-inf $W$, il n'existe aucune différence entre phrases libres et phrases figées au regard de la passivation. La passivation de ces phrases, avec placement de $N_{1}$ en position sujet, est acceptée avec même degré d'acceptabilité que la passivation des phrases $N_{0}$ faire $V^{1}$-inf $W$ lorsque $V$-inf est un inaccusatif - ce qui confirme l'identité de traitement pour $N_{1}$ sujet d'un verbe inaccusatif et $N_{1}$ complément d'objet direct d'un verbe transitif :

On a fait boire du lait à Max

= ?Du lait a été fait boire à Max

On a fait avaler la pilule à Max

$=\quad$ ?La pilule a été faite avaler à Max

On rappelle pour mémoire qu'en italien, en occitan et en français jusqu'au 17 ème siècle la passivation est pleinement acceptée pour les phrases de structure $\mathrm{N}_{0}$ faire $\mathrm{V}^{2}$ $\inf W$ : 
Italien

Hanno fatto stampare questo documento a spese dello statu

= Questo documento è stato fatto stampare a spese dello stato

Occitan

An fach estampar aquel document als fraisses del estat

$=\quad$ Aquel document es estat fach estampar als fraisses del estat

Français classique

On a fait amener Jean Ricous en une salle du chateau de l'Arsenal

$=\quad$ A été fait amener en une salle du château de l'Arsenal Jean Ricous ${ }^{10}$

\subsection{Les phrases figées de structure $N$ o faire $V$-inf $W$ par $N$}

Les phrases figées de structure $N_{0}$ faire $V$-inf $W$ par $N$ sont comparables en nombre aux phrases figées de structure $N_{0}$ faire $V^{2}$-inf $W$ (29 sur les 132 de notre corpus). Elles relèvent de la construction 2b. Par N, «complément circonstanciel » pour Damourette et Pichon qui lui assignent pour rôle celui d'« agent d'exécution » est noté dans la colonne $A d v$. La plupart du temps, il est non exprimé. Sont des phrases $N_{0}$ faire $V$-inf $W$ par $N$ les phrases notées «- » dans la colonne $V^{1}$-inf $W$ (i.e. les phrases dont le sujet de $V$-inf n'est pas $N_{1}$ ) et notées «- » également dans la colonne $V^{2}$-inf $W$ (i.e. les phrases dont le sujet n'est pas non plus $N_{2}$ ). Le sujet ne peut donc être que le $G N$ exprimé ou susceptible d'être exprimé par le complément en par.

Dans l'ensemble de ces phrases, l'opérateur causatif $N_{0}$ faire \# joue deux rôles différents : soit il garde son rôle d'opérateur causatif, comme dans la phrase libre Max s'est fait arracher une dent, soit il sert à topicaliser un actant complément de la phrase régie par l'opérateur causatif, en conférant à la phrase figée une sorte de voix passive, comme dans la phrase libre Max s'est fait écraser par une voiture ou Max s'est fait piquer par un serpent. Dans les phrases suivantes, $N_{0}$ faire \# est un opérateur causatif fonctionnant comme tel :

$$
\begin{aligned}
& \text { Max se fait mal voir } \\
& \text { Max se fait oublier } \\
& \text { Max se fait prier } \\
& \text { Max s'est fait porter pâle } \\
& \text { etc. }
\end{aligned}
$$

$N_{0}$ est l'agent instigateur ou responsable du processus décrit dans la proposition élémentaire (être mal vu, être oublié, être prié, être porté pâle, etc.). Dans les phrases suivantes $N_{0}$ faire \# est un opérateur causatif à fonction de topicalisation :

$$
\begin{aligned}
& \text { Max s'est fait enguirlander } \\
& \text { Max s'est fait trouer la peau }
\end{aligned}
$$

${ }^{10}$ Interrogatoire de Bertauld et Ricous. Samedi 11 octobre 1653. Mémoires de Mademoiselle, t. II, p. 556, in Damourette et Pichon, III, $\$ 1102$. 


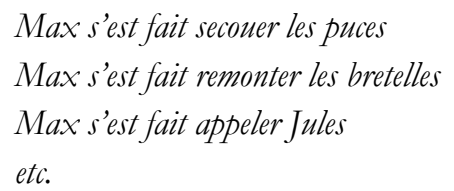

$N_{0}$ a un rôle de patient ou de bénéficiaire, celui-là même du C.O.D. ou du C.O.I. de la phrase élémentaire - représenté par $s e-$ et auquel $N_{0}$ est coréférent. Notons en outre que ces phrases, quoique moins courantes que les phrases correspondantes à opérateur causatif, sont généralement admises, avec le même sens :

\section{?On l'a enguirlandé, Max \\ On lui a troué la peau, à Max \\ On lui a secoué les puces, à Max \\ On lui a remonté les bretelles, à Max \\ ?On l'a appelé Jules, Max}

L'opérateur causatif à fonction de topicalisation peut être considéré comme une sorte d'opérateur à lien (M. Gross 1981). Comme tout opérateur à lien, il exige en effet un rapport de coréférence entre son sujet et un GN complément de la phrase élémentaire, et l'effet sémantique obtenu est de faire de ce GN de la phrase complément le point de départ et le centre d'intérêt de l'énoncé, autrement dit son topique. Rappelons le fonctionnement de l'opérateur à lien classique $N_{0}$ avoir \# :

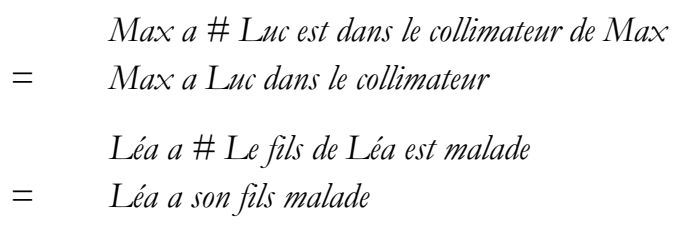

Ces emplois montrent que phrases élémentaires et phrases à opérateur causatif ont même sens, mais l'organisation de l'information est différente. Ainsi, dans les phrases élémentaires, il est question respectivement de Luc et du fils de Léa, tandis que dans les phrases à opérateur causatif à fonction de topicalisation, il est question respectivement de Max et de Léa, mis au premier plan.

\section{Conclusion}

L'analyse syntaxique des expressions causatives figées du français fait apparaître trois types de construction qui correspondent à ceux là même que distinguent Damourette et Pichon pour les phrases causatives libres :

- La construction directe, dans laquelle la phrase élémentaire est représentée par une proposition infinitive (cf. Paul le fait chanter une chanson, Paul le fait marcher).

- La construction à groupe verbal coalescent faire $V$-inf, dans laquelle le sujet de la phrase élémentaire est représenté par un complément datif (cf. Paul lui a fait chanter une chanson, Paul lui a fait sauter le caisson). 
- La construction à groupe verbal coalescent faire $V$-inf, dans laquelle le sujet de la phrase élémentaire est représenté par un complément en par/de (cf. Paul a fait chanter une chanson par Louis, Paul s'est fait oublier des autorités).

Dans le dernier type de construction, seule est attestée - pour les phrases figées la structure dans laquelle un complément de la phrase élémentaire est coréférent au sujet $N_{o}$ de l'opérateur causatif. Toute les structures observées sont donc en se faire $V$ inf. Cependant, pour les phrases figées comme pour les phrases libres, deux fonctions se distinguent clairement pour l'opérateur causatif : soit l'opérateur causatif joue son rôle classique d'opérateur causatif (le sujet $N_{0}$ de faire agit en sorte qu'un tiers exécute une action particulière ou se trouve dans une situation particulière) : Jean s'est fait soigner par un médecin, Jean s'est fait oublier de la police, soit l'opérateur causatif a une fonction de topicalisation pour un actant de la phrase élémentaire qu'il régit : Jean s'est fait piquer par un serpent, Jean s'est fait enguirlander par le patron.

Dans le cas des phrases libres, les trois types de construction sont en certains cas possibles, avec les nuances sémantiques signalées plus haut. Dans le cas des phrases figées, seule l'une des trois constructions est observable, ce qui permet de répartir l'ensemble des phrases en trois sous-ensembles naturels. Le caractère idiomatique de ces phrases et les spécificités de sens propres à chacune des constructions expliquent l'appartenance des phrases figées à une seule des trois constructions. 


\section{RÉFÉRENCES BIBLIOGRAPHIQUES}

BoOns, J.-P., Guillet, A., LeClÈre, C. (1976), La structure des phrases simples en français, Genève, Droz.

Bras, M. et Molinier, Ch. (2004), Locutions verbales en far de l'occitan. Constitution d'une base de données syntaxique, Cabiers de Grammaire, XX, Toulouse, ERSS-Université de Toulouse-Le Mirail.

Damourette, J. et PiCHOn, E. (1911-1950), Des mots à la pensée. Essai de Grammaire de la langue française, Paris, Editions d'Artrey.

GIRY-SCHNEIDER, J. (1978), Les nominalisations en français, Genève, Droz.

GIRY-SCHNEIDER, J. (1986), Les noms construits avec faire: compléments ou prédicats ? in Langue française, 69, Paris, Larousse.

GIRY-SCHNEIDER, J. (1987), Les prédicats nominaux en français, Genève, Droz.

Gross, M. (1975), Méthodes en syntaxe, Paris, Hermann.

Gross, M. (1981), Les bases empiriques de la notion de prédicat sémantique, Langages, 63, Paris, Larousse.

Gross, M. (1984), Une classification des phrases figées du français, in De la syntaxe à la pragmatique, P. Attal \& C. Muller (éds.), Amsterdam, John Benjamins B V.

Gross, M. (1986), Grammaire transformationnelle du français -Syntaxe du verbe, Paris, Larousse.

Gross, M. (1988), La phrase élémentaire et ses composants. Une discussion de quelques exemples, Travaux de Linguistique, 170, Paris-Gembloux, Duculot.

HAASE, A. (1965), Syntaxe française du XVII siècle, Paris, Delagrave.

Herslund, M. (1988), Le datif en français, Bibliothèque de l'Information grammaticale 14, Louvain-Paris, Editions Peeters.

KAYNE, R. (1977), Syntaxe du français. Le cycle transformationnel, Paris, Editions du Seuil.

Molinier, Ch. et LeVrier, F. (2000), Grammaire des adverbes, Genève, Droz.

Morin, Y.-Ch. (1980), Les bases syntaxiques des règles de projection sémantique: l'interprétation des constructions en faire, Linguisticae Investigationes 4, Amsterdam, John Benjamins B V.

Rey, A. et Chantreau, S. (1979), Dictionnaire des expressions et locutions figurées, Paris, Les usuels du Robert.

Ronjat, J. (1937), Grammaire historique des parlers provençaux modernes, I, II, III (III syntaxe), Montpellier, Société des langues romanes.

Rouveret, A. et Vergnaud, J.-R. (1980), Specifying reference to the subject: French causatives and conditions of representation, Linguistic Inquiry 11, Cambridge, MIT Press.

Ruwet, N. (1982), Grammaire des insultes et autres études, Paris, Editions du Seuil. 
Sur les constructions causatives figées du français

TAsmowski-De RYCK, L. (1985), Faire infinitif, in Melis, L., Tasmowski, L., Verluyten, P., Willems, D. (éds), Les constructions de la phrase simple, Ghent, Communication and Cognition.

Vaugelas, Cl. Favre de, (1647), Remarques sur la langue française (J. Streicher, fac-simile de l'édition de 1647, Paris, Droz, 1934). 
TABLE FV

\begin{tabular}{|c|c|c|c|c|c|c|c|c|c|c|}
\hline \multicolumn{2}{|c|}{$\mathrm{N}_{0}$} & \multirow[t]{2}{*}{$\mathrm{V}$} & \multicolumn{3}{|r|}{$V$-inf } & \multirow[t]{2}{*}{$\mathrm{N}_{1}$} & \multirow{2}{*}{ 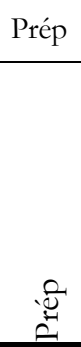 } & \multicolumn{2}{|l|}{$\mathrm{N}_{2}$} & \multirow[t]{2}{*}{ Adv } \\
\hline 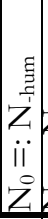 & 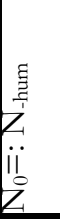 & & 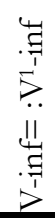 & 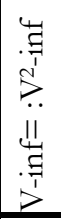 & & & & $\begin{array}{l}\text { 罢 } \\
\text { ZZ } \\
\text { iI } \\
\text { Z }\end{array}$ & $\begin{array}{l}\text { 䛼 } \\
\text { Z } \\
\text { iI } \\
\text { Z }\end{array}$ & \\
\hline+ & - & en faire & - & + & accroire & & à & + & - & \\
\hline+ & - & en faire & - & + & baver & & à & + & - & \\
\hline+ & - & en faire & + & - & passer & quinze & & & & $\begin{array}{l}\text { pour } \\
\text { douze }\end{array}$ \\
\hline+ & - & en faire & - & + & voir & & à & + & - & \\
\hline+ & - & en faire & - & + & voir & $\begin{array}{l}\text { de toutes les } \\
\text { couleurs }\end{array}$ & à & + & - & \\
\hline+ & - & faire & - & + & accroire & $\mathrm{N}_{\mathrm{nr}}$ & à & + & - & \\
\hline+ & - & faire & + & - & aller & $\mathrm{E}$ & & & & \\
\hline- & + & faire & + & - & aller & N-hum & & & & à la selle \\
\hline- & + & faire & + & - & aller & N-hum & & & & \\
\hline+ & - & faire & + & - & aller & N-hum & & & & \\
\hline+ & - & faire & + & - & aller & N-hum & & & & \\
\hline+ & - & faire & - & + & avaler & la pilule & à & + & - & \\
\hline+ & - & faire & - & + & avaler & $\mathrm{N}_{\mathrm{nr}}$ & à & + & - & \\
\hline+ & - & faire & - & + & baisser & la canterelle & $\overline{\mathrm{a}}$ & + & - & \\
\hline+ & - & faire & + & - & battre & des montagnes & & & & \\
\hline+ & + & faire & + & - & battre & le coeur & à & + & - & \\
\hline+ & - & faire & + & - & bouillir & la marmite & & & & \\
\hline+ & - & faire & + & - & bouillir & le pot & & & & \\
\hline+ & - & faire & + & - & bruire & Poss $_{0}$ fuseaux & & & & \\
\hline+ & - & faire & + & - & chanter & N-hum & & & & \\
\hline+ & - & faire & - & + & chanter & $\begin{array}{l}\text { une autre chan- } \\
\text { son }\end{array}$ & à & + & - & \\
\hline+ & - & faire & + & - & chauffer & la colle & & & & \\
\hline+ & + & faire & + & - & chier & N-hum & & & & \\
\hline- & + & faire & + & - & couler & \begin{tabular}{|l|} 
beaucoup \\
d'encre
\end{tabular} & & & & \\
\hline- & + & faire & + & - & couler & $\begin{array}{l}\text { beaucoup de } \\
\text { salive }\end{array}$ & & & & \\
\hline+ & - & faire & + & - & damner & un saint & & & & \\
\hline+ & - & faire & + & - & danser & l'anse du panier & & & & \\
\hline+ & - & faire & - & + & danser & la Carmagnole & à & + & - & \\
\hline+ & - & faire & + & - & danser & N-hum & & & & \\
\hline+ & - & faire & + & - & danser & N-hum & & & & \begin{tabular}{|l|} 
sans \\
violons
\end{tabular} \\
\hline
\end{tabular}




\begin{tabular}{|c|c|c|c|c|c|c|c|c|c|c|}
\hline+ & - & faire & + & - & donner & la troupe & & & & \\
\hline+ & - & faire & + & - & donner & le canon & & & & \\
\hline+ & + & faire & + & - & dresser & les cheveux & à & + & - & $\begin{array}{l}\text { sur la } \\
\text { tête }\end{array}$ \\
\hline+ & - & faire & + & - & durer & le plaisir & & & & \\
\hline+ & - & faire & + & - & enrager & N-hum & & & & \\
\hline+ & - & faire & - & + & entendre & Poss $_{0}$ voix & à & + & - & \\
\hline+ & - & faire & - & + & entendre & raison & $\bar{a}$ & + & - & \\
\hline+ & - & faire & + & - & gémir & la presse & & & & \\
\hline- & + & faire & + & - & jaser & $\mathrm{E}$ & & & & \\
\hline+ & - & faire & + & - & jouer & la corde sensible & & & & \\
\hline+ & - & faire & + & - & marcher & le ban & & & & \\
\hline- & + & faire & + & - & marcher & le commerce & & & & \\
\hline+ & - & faire & + & - & marcher & N-hum & & & & \\
\hline+ & - & faire & + & - & marcher & N-hum & & & & \\
\hline+ & + & faire & + & - & marrer & N-hum & & & & \\
\hline+ & + & faire & + & - & mentir & le proverbe & & & & \\
\hline+ & - & faire & + & - & mourir & N-hum & & & & $\begin{array}{l}\text { à petit } \\
\text { feu }\end{array}$ \\
\hline+ & - & faire & + & - & parler & $\mathrm{E}$ & & & & de $\mathrm{LUI}_{0}$ \\
\hline+ & + & faire & + & - & passer & l'enfant & & & & \\
\hline+ & + & faire & + & - & passer & le goût du pain & à & + & - & \\
\hline+ & - & faire & - & + & passer & $\begin{array}{l}\text { un mauvais } \\
\text { quart d'heure }\end{array}$ & à & + & - & \\
\hline+ & + & faire & + & - & pencher & la balance & & & & \\
\hline+ & + & faire & + & - & penser & N-hum & à & + & + & \\
\hline+ & - & faire & - & + & perdre & le goût du pain & à & + & - & \\
\hline+ & + & faire & + & - & périr & N-hum & & & & \\
\hline+ & + & faire & + & - & périr & N-hum & & & & \\
\hline+ & - & faire & - & + & porter & des cornes & à & + & - & \\
\hline+ & - & faire & + & - & pousser & N-hum & & & & \\
\hline+ & - & faire & + & - & rentrer & les mots & à & + & - & $\begin{array}{l}\text { dans la } \\
\text { gorge }\end{array}$ \\
\hline+ & - & faire & + & - & rire & un tas de pierres & & & & \\
\hline+ & - & faire & + & - & sauter & la cervelle & à & + & - & \\
\hline+ & - & faire & + & - & sauter & le bouchon & & & & \\
\hline+ & - & faire & + & - & sauter & le caisson & à & + & - & \\
\hline+ & - & faire & + & - & sauter & les scellés & & & & \\
\hline+ & - & faire & + & - & sauter & N-hum & & & & $\begin{array}{l}\text { sur } \operatorname{Poss}_{0} \\
\text { genoux }\end{array}$ \\
\hline+ & - & faire & + & - & sonner & Poss $_{0}$ grelot & & & & \\
\hline+ & - & faire & + & - & suer & le bonhomme & & & & \\
\hline+ & - & faire & + & - & suer & le burnous & & & & \\
\hline+ & + & faire & + & - & suer & N-hum & & & & \\
\hline+ & - & faire & + & - & toucher & les épaules & à & + & - & \\
\hline+ & - & faire & + & - & tourner & la tête & à & + & - & \\
\hline
\end{tabular}




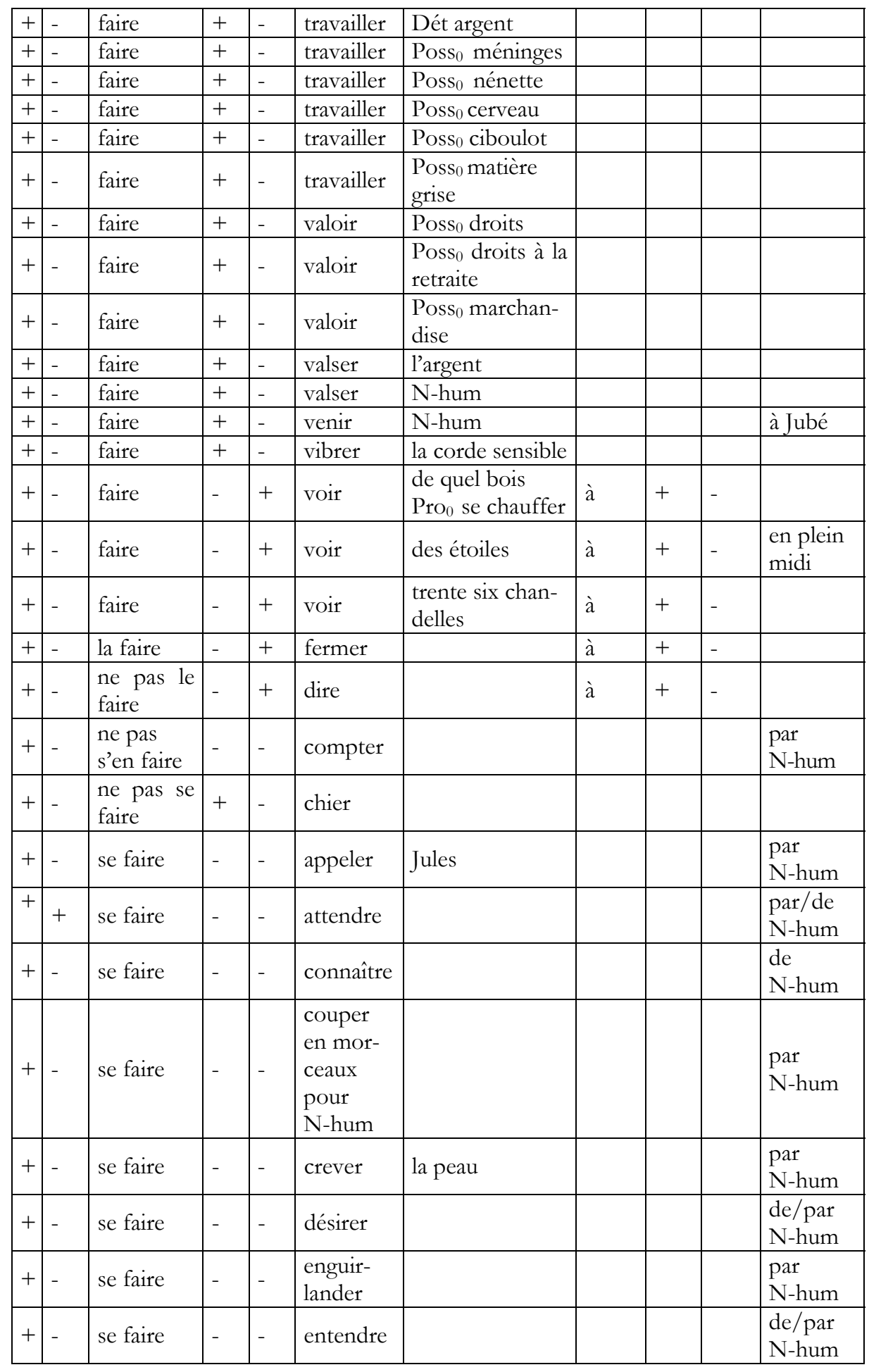


Sur les constructions causatives figées du français

\begin{tabular}{|c|c|c|c|c|c|c|c|c|c|}
\hline+ & - & se faire & - & - & $\begin{array}{l}\text { hacher en } \\
\text { morceaux } \\
\text { pour } \\
\text { Nhum }\end{array}$ & & & & $\begin{array}{l}\text { par } \\
\text { N-hum }\end{array}$ \\
\hline+ & - & se faire & - & - & mal voir & & & & \begin{tabular}{|l} 
de \\
N-hum
\end{tabular} \\
\hline+ & - & se faire & + & - & mousser & & & & \\
\hline+ & - & se faire & - & - & oublier & & & & \begin{tabular}{|l} 
de \\
N-hum
\end{tabular} \\
\hline+ & - & se faire & - & - & $\begin{array}{l}\text { porter } \\
\text { pâle }\end{array}$ & & & & $\begin{array}{l}\text { par } \\
\text { N-hum }\end{array}$ \\
\hline+ & - & se faire & - & - & prier & & & & $\begin{array}{l}\text { par } \\
\text { N-hum }\end{array}$ \\
\hline+ & - & se faire & - & - & $\begin{array}{l}\text { raccour- } \\
\text { cir }\end{array}$ & & & & $\begin{array}{l}\text { par } \\
\text { N-hum }\end{array}$ \\
\hline+ & - & se faire & - & - & ramasser & & & & $\begin{array}{l}\text { par } \\
\text { N-hum }\end{array}$ \\
\hline+ & - & se faire & - & - & recevoir & & & & $\begin{array}{l}\text { par } \\
\text { N-hum }\end{array}$ \\
\hline+ & - & se faire & - & - & remarquer & & & & $\begin{array}{l}\text { par } \\
\text { N-hum }\end{array}$ \\
\hline+ & - & se faire & - & - & remonter & les bretelles & & & $\begin{array}{l}\text { par } \\
\text { N-hum }\end{array}$ \\
\hline+ & - & se faire & - & - & rincer & & & & $\begin{array}{l}\text { par } \\
\text { N-hum }\end{array}$ \\
\hline+ & - & se faire & + & - & sauter & le plafond & & & \\
\hline+ & - & se faire & - & - & secouer & les puces & & & \begin{tabular}{|l} 
par \\
N-hum
\end{tabular} \\
\hline- & + & se faire & - & + & sentir & & à & + & - \\
\hline+ & - & se faire & - & - & tirer & l'oreille & & & \begin{tabular}{|l} 
par \\
N-hum
\end{tabular} \\
\hline+ & - & se faire & - & - & tirer & les vers du nez & & & $\begin{array}{l}\text { par } \\
\text { N-hum }\end{array}$ \\
\hline+ & - & se faire & - & - & tondre & & & & $\begin{array}{l}\text { par } \\
\text { N-hum }\end{array}$ \\
\hline+ & - & se faire & - & - & tondre & $\begin{array}{l}\text { la laine sur le } \\
\text { dos }\end{array}$ & & & \begin{tabular}{|l|} 
par \\
N-hum
\end{tabular} \\
\hline+ & - & se faire & - & - & trouer & la peau & & & \begin{tabular}{|l|} 
par \\
N-hum
\end{tabular} \\
\hline+ & - & se faire & - & - & $\begin{array}{l}\text { tuer } \\
\text { jusqu'au } \\
\text { dernier }\end{array}$ & & & & $\begin{array}{l}\text { par } \\
\text { N-hum }\end{array}$ \\
\hline+ & - & se faire & - & - & voir & & & & $\begin{array}{l}\text { par/de } \\
\text { N-hum }\end{array}$ \\
\hline+ & - & se faire & - & - & $\begin{array}{l}\text { voir sous } \\
\text { Poss }_{0} \\
\text { meilleur } \\
\text { aspect }\end{array}$ & & & & $\begin{array}{l}\text { par/de } \\
\text { N-hum }\end{array}$ \\
\hline
\end{tabular}


Christian Molinier

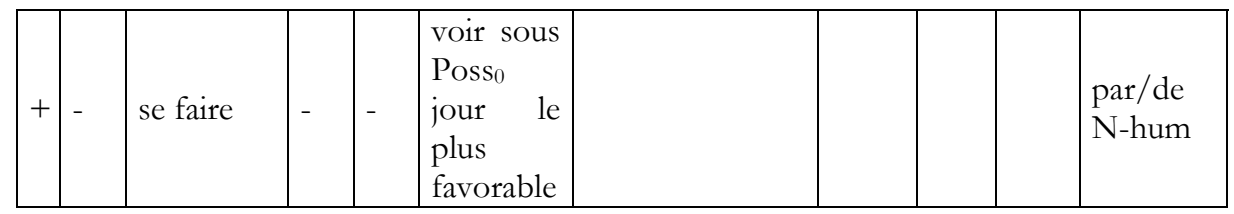

